\title{
COMPORTAMENTO DE DIVERSAS PLANTAS DANINHAS, DE OCORRÊNCIA COMUM NO ESTADO DE SÃO PAULO, EM RELAÇÃO A DUAS ESPÉCIES DE NEMATÓIDES DAS GALHAS; PRIMEIRA PARTE
}

\author{
L.C.C.B. FERRAZ ${ }^{1}$ \\ 1 ESALQ-USP - Piracicaba - \\ Departamento de Zoologia.
}

\section{RESUMO}

Estudou-se o comportamento de 10 espécies de plantas daninhas,co muns no Estado de São Paulo, quando o solo em que vegetavam foi inoculado com os nematóides das galhas Meloidogyne incognita raça 4 ou M. javanica. As avaliações foram feitas 50 dias após a inoculação dos parasitos, baseando-se nos números de galhas e ootecas pre sentes nas raízes e nos valores de altura e de pesos secos da parte aérea e sistemas radiculares das plantas. Em relação as duas espécies de nematóides, comportaram-se como alta mente suscetíve is Alternaria ficoidea (apaga-fogo) e Ipomoea acuminata (cordade-viola), como olerantes Amaranthus hybridus var. patulus (caruru) e Commelina virgunica (trapoeraba), como pouco suscetível Euphorbia heterophylla (amendoim bravo) e como altamente resistentes Blainvillea rhomboidea (ervapalha), Crotonn glandulosus (gervãobranco), Emilia sonchifolia (serralha) e Tagetes minuta (cravo-de-defunto). O carrapicho-de-carneiro, Acanthorpermum hispidum, mostrou-se altamente resis tente a $M$. incognita raça 4 e moderadamente suscetível a $M$. javanica.
PALAVRAS-CHAVE: plantas daninhas hospedeiras, Meloidogyne.

\section{SUMMARY}

The behaviour of ten different weed species belonging to Amaranthaceae, Commelinaceae, Compositae, Convolvulaceae and Euphorbiaceae in relation to the root-knot nematodes Meloidogyne incognita race 4 and $M$. javanica was studied under greenhouse conditions. Evaluations were carried out 50 days after the nematode inoculations, by means of gall and egg masses indexes observed in the root systems and through the determination of height and/or top dry weight of the plants. Alternanthera ficoidea and Ipomoea acuminate were considered highly suscetible to both nematode species; Amaranthus hybridus var. patulus and Commelina virgunica were tolerant to the arasites whereas Euphorbia heterophylla showed low susceptibility; Acanthospernum hispidum was considered moderately suscetible to $\mathrm{M}$. javanica and highly resistant to $M$. incognita race 4; all other weeds - Blainvillea rhomboidea, Cro- 
ton glandulosus, Emilia sonchifolia and Tagetes minuta - were highly resistant to the nematodes.

KEY-WORDS: weed hosts, root-knot nematodes.

\section{INTRODUÇÃO}

As plantas daninhas competem diretamente com as espécies vegetais cultivadas por fatores de crescimento como água, luz, espaço e nutrien tes. Além disso, dentre outras, podem servir de hospedeiras a fitone matóides, assegu rando-lhes a sobrevi

No Brasil já existem diversos relatos de associações entre plantas daninhas e nematóides fitoparasitos, particularmente os pertencentes ao gênero Meloidogyne (for madores de galhas), sumarizados por Ferraz et al. (1983). Todavia, com algumas exceções, como o de Antonio \& Dall'Agnol (1983), a maioria dos trabalhos limita-se apenas a incluir as plantas daninhas no rol de hospedeiros de diferentes espécies de nematói des.

No presente estudo, objetivou-se avaliar o comportamento de diversas plantas daninhas pertencentes às famílias Amaran thaceae, Commelinaceaea, Compositae, Convolvulaceae e Euphorbiaceae, comumente encontradas no Estado de São Paulo, quando inoculadas com os nematóides das galhas Meloidogyne incognita (Kofoid \& White, 1919) Chit-wood, 1949 raça 4 ou $M$. javanica (Treub, 1885) Chitwood, 1949, verificando-se os graus de infestação dos parasitos e a ocorrência ou não de re dução no crescime nto da s pl an tas.

\section{MATERIAL E MÉTODOS}

As plantas daninhas utilizadas no trabalho foram as seguintes, a presentadas segundo a posição sis temática e nomes populares propostos por Lorenzi (1982): Ama ranthaceae - Alternanthera ficoidea (L.) Br. (apaga-fogo) e Amaranthus hybridus var. patulus Thell. (caruru); Commelinaceae - Commelina virginica L. (trapoe raba); Campostitae

Acanthospermun hispidium DC. (carrapicho-de-carneiro), Blainvillea rhomboidea Cass. (erva-palha), Emilia sonchifolia (serralha) e Tagetes minuta L. (cravo-de-defunto); Convolvulaceae Ipomoea acuminata Roem. et Sch. (cordade-viola); Euphorbiaceae - Croton glandulosus (L.) Muell. (gervão-branco) e Euphorbia heterophylla L. (amendoimbravo).

O delineamento estabelecido foi de blocos casualizados com 3 tratamentos (inoculado com Metoidogyne incognita, inoculado com $M$. javanica e testemunha não inoculada) e 7 repetições para cada espécie de planta daninha.

As plantas foram inicialmente conduzidas em copos plásticos de $250 \mathrm{ml}$ contendo solo esteri liza do, cons erv ando-se uma por recipiente após o desbaste. Posteriormente, for am transplantadas para vasos com 3,5 litros de solo, também esterilizado, mantidos em cas a de vegetação.

A inoculação foi feita 24 a 48 horas após o transplante, liberando-se 28.000 ovos de $M$. incognita ou de $M$. iavanica por vaso $(8.000$ ovos/litro de solo) através de funil de vidro dirigido para o interior de 3 orifícios abertos ao 
redor de cada planta, atingindo-se assim a região da rizosfera. Cinqüenta dias após a inoculação, as plantas foram cuidadosamente arrancadas, tiveram as raízes bem lavadas e foram levadas ao laboratório para as avaliações previstas.

Os sistemas radiculares foram avaliados quanto aos números de galhas (método 1) e de ootecas (método 3), adotando-se a escala de notas de Taylor \& Sasser (1978), variável de 0 a 5. Utilizou-se ainda um terceiro método baseado na porcentagem do sistema radicular que se apresentava com galhas, designado como método 2 , empregando-se a seguinte escala de notas: nota $0=0 \%$ do sistema radicular com galhas; nota $1=$ entre 0,1 a $10 \%$ do sistema radicular com galhas; nota $2=$ entre 10,1 e $25 \%$ do sistema radicular com galhas; nota $3=$ entre 25,1 e $50 \%$ do sistema radicular com galhas; nota 4 $=$ entre $50,175 \%$ do sistema radicular com galhas; e nota $5=$ mais de $75 \%$ do sis tema radicular com galhas.

Após as avaliações, as raízes foram colocadas em sacos de papel e postos para secar em estufa à $65-68^{\circ} \mathrm{C}$, até peso constante. Nos casos de algumas plantas daninhas, a cujos sistemas radiculares atribuiu-se preponderantemente a nota zero, determinaram-se os números de nematóides (larvas infestantes) por $10 \mathrm{~g}$ de raízes ao invés dos pesos secos, sendo os espécimes obtidos pelo método combinado do liquidificador e peneiramento.

Em relação à parte aérea, procedeu-se inicialmente à medição da altura das plantas de caule praticamente ereto, considerada como sendo a distância, no caule, do colo até a última ramificação lateral. Posteriormente, os órgãos aéreos foram reduzidos a pequenos pedaços e levados para secar em estufa do mesmo modo que os sistemas radiculares. Os dados de altura e pesos secos foram submetidos ao teste $\mathrm{F}$ e as médias, sempre que viável, comparadas pelo teste de Tukey.

A análise do comportamento baseou-se no critério proposto por Dropkin \& Nelson (1960), classificando-se as espécies vegetais em suscetíveis, resistentes, tolerantes ou intolerantes.

\section{RESULTADOS E DISCUSSÃO}

Os resultados obtidos (valores médios das 7 repetições) para grau de infestação dos sistemas radiculares pelos 3 métodos de avaliação, peso seco da parte aérea, peso seco de raízes, altura das plantas e número de larvas por $10 \mathrm{~g}$ de raízes estão apresentados no Quadro 1.

Pelos valores determinados, verifica-se que, dentro do grupo de plantas estudadas, ocorreram desde espécies altamente susce tíveis, até muito resistentes.

A corda-de-viola (I. acuminata) foi a planta daninha cujos sistemas radiculares receberam maiores notas em relação às presenças de galhas e ootecas, apresentandose deformados e atrofiados. Essa espécie, juntamente com o apaga-fogo (A. ficoidea) foram consideradas muito suscetíveis aos nematóides inoculados, observando-se diferenças significativas entre os pesos secos da parte aérea e das raízes dos tratamentos inoculados em relação à testemunha.

$\mathrm{O}$ caruru (A. hybridus var. patulus) sofreu infestações preponderantemente moderadas, embora algumas plantas mostrassem ataques severos. As galhas eram bastante evidentes e ocorriam tanto ootecas externas como internas. Como não se 
Quadro 1. Valores médios (7 repetições) de graus de infestação dos sistemas radiculares, avalia dos por 3 diferentes métodos, pesos secos da parte aérea e das raízes (em gramas), al tura das plantas (em centímetros) e no de larvas por $10 \mathrm{~g}$ de raízes determinados para as espécies daninhas estudadas.

\begin{tabular}{|c|c|c|c|c|c|c|c|c|c|c|c|}
\hline & & $\begin{array}{l}\text { A1 thernan thern } \\
\text { f10o1don }\end{array}$ & $\begin{array}{l}\text { Amarastbus } \\
\text { hybridus }\end{array}$ & $\begin{array}{l}\text { Comoliten } \\
\text { virgialen }\end{array}$ & $\begin{array}{l}\text { Aennshosporeuse } \\
\text { bispidua }\end{array}$ & $\begin{array}{c}\text { Bla10rillea } \\
\text { ragmboldes }\end{array}$ & $\begin{array}{c}\text { Intlia } \\
\text { osnehtronia }\end{array}$ & $\begin{array}{l}\text { Tagetes } \\
\text { glauta }\end{array}$ & $\begin{array}{c}\text { Iponoses } \\
\text { meuslazta }\end{array}$ & $\begin{array}{c}\text { Croteo } \\
\text { slacosuzinas } \\
\end{array}$ & $\begin{array}{l}\text { Iushortid } \\
\text { heterophy11: }\end{array}$ \\
\hline \multirow{2}{*}{ 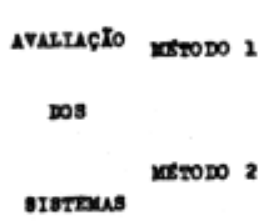 } & $\begin{array}{l}\text { L. Ioosgnita } \\
\text { M. faranica }\end{array}$ & $\begin{array}{l}2,7 \\
2,86\end{array}$ & $\begin{array}{l}3,29 \\
3,14\end{array}$ & $\begin{array}{l}2,86 \\
3,14\end{array}$ & $\begin{array}{l}0,00 \\
3,29\end{array}$ & $\begin{array}{l}0,86 \\
0,00\end{array}$ & $\begin{array}{l}0,86 \\
0,29\end{array}$ & $\begin{array}{l}0, \infty \\
0, \infty\end{array}$ & $\begin{array}{l}4,00 \\
3,85\end{array}$ & $\begin{array}{l}1, c 0 \\
0,57\end{array}$ & $\begin{array}{l}3,43 \\
2,86\end{array}$ \\
\hline & $\begin{array}{l}\text { M. Lacogalta } \\
\text { x. Javanion }\end{array}$ & $\begin{array}{l}3,86 \\
3,57\end{array}$ & $\begin{array}{l}3,00 \\
3,43\end{array}$ & $\begin{array}{l}1, \infty \\
1, \infty\end{array}$ & $\begin{array}{l}0, \infty \\
2,14\end{array}$ & $\begin{array}{l}0,57 \\
0,00\end{array}$ & $\begin{array}{l}0,57 \\
0,29\end{array}$ & $\begin{array}{l}0, \infty \\
0, \infty\end{array}$ & $\begin{array}{l}4,57 \\
4,29\end{array}$ & $\begin{array}{l}0, \pi 1 \\
0,43\end{array}$ & $\begin{array}{l}2,43 \\
1,14\end{array}$ \\
\hline uproulases ntiom 3 & $\begin{array}{l}\text { x. Luoogalta } \\
\text { M. Javanics }\end{array}$ & $\begin{array}{l}2,86 \\
2,06\end{array}$ & $\begin{array}{l}3,14 \\
3,43\end{array}$ & $\begin{array}{l}2,57 \\
2,71\end{array}$ & $\begin{array}{l}0,00 \\
2,57\end{array}$ & $\begin{array}{l}0,00 \\
0,00\end{array}$ & $\begin{array}{l}0,29 \\
0,00\end{array}$ & $\begin{array}{l}0,00 \\
0, \infty\end{array}$ & $\begin{array}{l}4,00 \\
3,71\end{array}$ & $\begin{array}{l}0,29 \\
0,00\end{array}$ & $\begin{array}{l}1,29 \\
1,00\end{array}$ \\
\hline \multirow{2}{*}{ 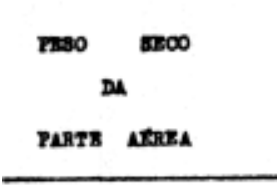 } & $\begin{array}{l}\text { Y. Inoogaits } \\
\text { R. Javaolea } \\
\text { Tostenuaba }\end{array}$ & $\begin{array}{l}0,73 \mathrm{~b} \\
0,81 \mathrm{~b} \\
2,30 \mathrm{a}\end{array}$ & $\begin{array}{l}2,90 \\
2,86 \\
2,95 \\
\end{array}$ & $\begin{array}{l}0,03 \\
0,03 \\
0,03 \\
\end{array}$ & $\begin{array}{l}16,25 \\
15,24 \\
15,68 \\
\end{array}$ & $\begin{array}{l}4,56 \\
4,54 \\
4,52 \\
\end{array}$ & $\begin{array}{l}1,65 \\
1,63 \\
1,68 \\
\end{array}$ & $\begin{array}{l}4,70 \\
4,74 \\
4,66 \\
\end{array}$ & $\begin{array}{l}0,10 \mathrm{~b} \\
0,12 \mathrm{~b} \\
0,39 \mathrm{a}\end{array}$ & $\begin{array}{l}1,15 \\
1,11 \\
1,17\end{array}$ & $\begin{array}{l}0,74 \\
0,72 \\
0,82\end{array}$ \\
\hline & $F$ trat. & $136,88^{\circ}$ & 0,1900 & 0,3500 & 2,14 ne & $0,09 \mathrm{~ns}$ & $0,38 \mathrm{a}$ & $0,03 \mathrm{ab}$ & $107,10^{\circ}$ & 0,78 a & 1,21 as \\
\hline \multirow[t]{2}{*}{$\begin{array}{c}\text { Feso } \\
\text { nus } \\
\text { nufzes }\end{array}$} & $\begin{array}{l}\text { I. Inesgalta } \\
\text { M. Jevanios } \\
\text { Teotenunba }\end{array}$ & $\begin{array}{l}0,11 \text { b } \\
0,12 b \\
0,35=\end{array}$ & $\begin{array}{l}0,36 \\
0,37 \\
0,37 \\
\end{array}$ & $\begin{array}{l}0,01 \\
0,01= \\
0,01 \\
\end{array}$ & $\begin{array}{l}1,11 \\
0,98 \\
1,07 \\
\end{array}$ & $\begin{array}{l}- \\
- \\
-\end{array}$ & $\begin{array}{l}0,33 \\
0,32 \\
0,32\end{array}$ & $\begin{array}{l}- \\
- \\
-\end{array}$ & $\begin{array}{l}0,06 \mathrm{~b} \\
0,07 b \\
0,16\end{array}$ & $\begin{array}{l}0,20 \\
0,19 \\
0,21\end{array}$ & $\begin{array}{l}0,11 \\
0,11 \\
0,12 \\
\end{array}$ \\
\hline & $P$ tret. & $62,75^{\circ}$ & $0,26 \mathrm{no}$ & $0,22 \mathrm{ng}$ & $1,91 \mathrm{as}$ & & 0,4400 & & $50,75^{\circ}$ & $0,50 \mathrm{as}$ & 0,63 on \\
\hline \multirow{2}{*}{$\begin{array}{c}\text { ALruga } \\
\text { dus } \\
\text { rumtas }\end{array}$} & $\begin{array}{l}\text { y. Incsgalta } \\
\text { x. javanies } \\
\text { zostenuaba }\end{array}$ & - & $\begin{array}{l}39,06 \\
38,03 \\
39,23\end{array}$ & - & - & $\begin{array}{l}53,14 \\
54,20 \\
54,30\end{array}$ & $\begin{array}{l}35,19 \\
34,59 \\
34,90\end{array}$ & $\begin{array}{l}45,14 \\
44,34 \\
44,69\end{array}$ & - & $\begin{array}{l}30,30 \\
28,27 \\
29,89\end{array}$ & $\begin{array}{l}21,16 \\
22,04 \\
21,39\end{array}$ \\
\hline & P. trat. & & $1,13 \mathrm{ag}$ & & & $0,64 \mathrm{as}$ & 0,59 o & $0,57 \mathrm{as}$ & & 1,58 a & 0,48 o \\
\hline at larmed/10 rafses & $\begin{array}{l}\text { ‥ Ioeocalta } \\
\text { ‥ Javaniea }\end{array}$ & - & - & - & - & $\begin{array}{r}17,9 \\
8,9\end{array}$ & - & $\begin{array}{l}8,0 \\
3,4 \\
\end{array}$ & - & - & - \\
\hline
\end{tabular}


observaram diferenças significativas entre os diferentes tratamen tos relativamente aos pesos secos e altura das plantas, a espécie daninha em questão foi considerada tolerante aos nematóides.

A trapoeraba (C. virginica) teve as raízes moderadamente infestadas nas avaliações pelos métodos 1 e 3 . As galhas eram extremamente pequenas, sendo identificadas com segurança apenas ao estereomicros cópio. Além disso, durante o amadurecimento sexual, as fêmeas expunham a maior parte do corpo fora das raízes, praticamente descaracterizando os engrossamentos, o que determinou a atribuição de notas inferiores pelo método 2. Em relação ao crescimento, não ocorreram diferenças significativas entre as plantas inoculadas e não inoculadas. Tais observações e resultados mostraram-se concordantes com os apresentados por Antonio \& Dall'Agnol (1983) para $C$. virginica, tendo esses autores verificado, após 78 dias da inoculação, que a intensidade de infestação aumentava muito à medida que as plantas se tornavam mais velhas, mas sem caus ar prejuízo aparente no cres cimento. Consi derou-se, por tanto, com base nos dados ora obtidos e nas informações disponíveis, a trapoeraba como toler ante aos parasitos inoculados.

O amendoim-bravo (E. heterophylla) foi também infestado pelas 7 espécies de nematóides, prevalecendo ataques considerados moderados pelo método $1 \mathrm{e}$ leves pelo 2. As galhas eram geralmente pequenas e esparsas, porem, quando ocorriam agrupadas, orig in avam engrossamentos bem evidentes nas extremidades das radicelas. O número de ootecas foi sempre menor que o de galhas, sugerindo que muitos espécimes aind a não haviam completado o ciclo aos 50 dias após a inoculação. Por sua vez, isto pode indicar pouca atração das larvas às raízes ou relativa dificuldade no estabelecimento do parasitismo. Não ocorreram diferenças significativas entre as plantas dos 3 tratmentos em relação à altura e pesos secos de raízes ou parte aérea. Em vista do exposto, considerourse o amendoim-bravo hospedeiro pouco favorável, mostrando baixa suscetibilidade aos parastos. Essa espécie apresenta particular interesse como hospedeiro de Meloidogyne no Estado do Paraná, conforme ressaltado por Antonio \& Lehman (1978), dada sua grande disseminação e difícil controle em relação às culturas de soja locais.

No caso do carrapicho-de-carneiro ( $A$. hispidium), apenas M. javanica conseguiu parasitar as raizes, incitando o aparecimento de galhas pequenas nas extremadades das radicelas e formando ootecas somente visíveis com clareza ao estereomicroscópio. As infestações variaram de leves a moderadas, sendo estas as mais frequientes. Nos sistemas radiculares das plantas que receberam inoculo de $M$. incognita não ocorreram galhas ou ootecas, encontrando-se apenas esporádicas larvas pré-parasitas durante a dissecação das radicelas. O parasitismo de $M$. iavanica não causou redução no crescimento das plantas. A espécie em questão foi então considerada altamente resistente a $M$. incognta e moderadamente suscetível a $M$. javanica. Com referência a $M$. incognita, as presentes observações são totalmente concordantes com as de Rebel et al. (1974) e de Antonio \& Dall'Agnol (1983), embora tais autores não houvessem identificado a raça do nematóide. Em relação a M. javanica, Antonio \& Dal'Agnol 
(1983) consideraram A. hispidum hospedeiro bastante suscetível, tendo obtido valores mais altos para o número de ootecas que os do presente estudo, o que provavelmente se deveu aos diferentes períodos de observações (78 e 50 dias, respectivamente) adotados nos dois trabalhos.

As demais plantas daninhas estudadas erva palha, serralha, cravo-de-defunto e gervão branco - comportaram-se como hospedeiros muito desfavoráveis aos nematóides inoculados, predominando a atribuição de notas zero aos sistemas radiculares através dos 3 métodos de avaliação. Apenas em relação à serralha (E. sonchifolia) estabeleceu - se certa controvérsia, pois alguns autores (Ponte, 1968 e Ogbuji, 1978, por exemplo) incluíram-na anteriormente em listas de hospedeiros de $M$. incognita ou $M$. javanica, a partir de plantas coletadas durante levantamentos de campo. Uma possível explicação para essa variação observada no grau de suscetibilidade da serralha, e provavelmente de outras plantas daninhas, pode estar no fato de esses nematóides apresentarem várias raças, com diferentes círculos de hospedeiros. Em função do exposto, fica a sugestão de se examinar sempre os materiais de serralha obtidos nas condições locais, a fim de se estabelecer sua real importância como hospedeiro de nematóides das galhas.

De forma geral observa-se que, apesar da ampla variação ocorrida nos níveis de suscetibilidade ou resistência, cada uma das plantas daninhas mostrou comportamento praticamente uniforme em relação às 2 espécies de nematóides, excetuando-se apenas o carrapicho-de-carneiro que se mostrou muito resistente a $M$. incognita e moderada- mente suscetível à $M$. javanica.

Com referência aos diferentes métodos de avaliação da suscetibilidade ora utilizados, comentários serão apresentados na 24 parte do presente estudo, a qual envolvera a análise do comportamento de diversas plantas daninhas pertencentes a outras famílias botânicas também em relação aos nematóides das galhas.

\section{LITERATURA CITADA}

Intonio, H. \& A. Dall'Agnol. Susce tibilidade de plantas daninhas a 3 espécies de nematóides, segundo 3 métodos de avaliação. In: REUNIÃO BRASILEIRA DE NEMATOLOGIA, 7, Brasilia, 1983. Resumos, p.14.

Intonio, H. \& P.S. Lehman, Nota sobre a ocorrência de nematói des do gênero Meloidogyne em a $\overline{1}$ gumas ervas daninhas nos estados do Paraná e Rio Grande do Sul. Soc. Brasil. Nematol publ. 3: $29-32,1978$.

Jropkin, V.H. \& P.E. Nelson. The histopathology of root-knot nematode infections in soybeans. Phytopathology, 50: 442 - 447, 1960.

Ferraz, L.C.C.B.; R.A. Pitelli \& L.E. Bendixen. An annotated bi bliography of weeds as reser voirs for organisms affecting crops in Brazil. 1. Nematodes: Meloidogyne. Ohio State Univ. Res. Bul1. no 1153, 16p., 1983. Lorenzi, H. Plantas daninhas do Brasil. Nova Odessa (SP), (ed.) 425p., 1982.

Ogbuji, R.o. Weed hosts of Meloidogyne incognita in Nigeria. Ne matol. Hediterranea, 6: $229=$ 230, 1978.

Ponte, J.J. Subsidios ao conheci 
mento de plantas hospedeiras e ao controle dos nematóides das galhas, Meloidogyne spp., no Estado do Cearā. Bol. Cearense Agron., 9: 1-26, 1968.

Rebel, E.K.; L.G.E. Lorde11o \& $M$. V. Moraes. Plantas hospedei ras de um nematóide nocivo ao cafeeiro. Anais B.S.A. "Luiz de Queiroz", 31: 431 - 435, 1974.

raylor, A.L. \& J.N. Sasser. Biolo $B$, identification and control of root-knot nematodes. North Carolina State Univ. Graphies, Raleigh, USA, 11lp., 1978. 